\title{
Les expériences de vieillissement vécues par des femmes sénégalaises âgées de 60 ans et plus, vivant dans la communauté
}

\author{
Awa Seck, Ph.D.
}

École nationale de développement sanitaire et social (ENDSS)

Ginette Lazure, Ph.D.

\section{Université Laval}

Diane Morin, Ph.D.

\section{Université de Lausanne et Université Laval}

\begin{abstract}
Résumé
Cette étude vise à mieux comprendre la signification que des femmes sénégalaises âgées de 60 ans et plus donnent au vieillissement. Des entrevues non structurées auprès de dix participantes ont été analysées selon l'approche phénoménologique descriptive de Giorgi. Vingt-quatre sous-thèmes, huit thèmes et une structure générale émergent de l'analyse des données recueillies. Le vieillissement féminin est vécu comme un processus de réflexion existentielle et une expérience d'épanouissement malgré une vulnérabilité physique, psychologique et économique. Ainsi, pour ces femmes, vieillir représente une expérience paradoxale intégrant à la fois des satisfactions et des contraintes. Des recommandations ont été faites dans le sens de la mise en œuvre d'un programme de recherche visant l'amélioration des conditions de vie et de santé des femmes âgées de 60 ans et plus.

Mots clés

VIEILLISSEMENT, EXPÉRIENCES, FEMMES, PHÉNOMÉNOLOGIE, SÉNÉGAL
\end{abstract}

\section{Introduction}

Le vieillissement est un phénomène de plus en plus préoccupant dans les pays en développement où vivent plus de $60 \%$ des personnes vieillissantes de la planète (Organisation des Nations Unies (ONU), 2002). L'augmentation de cette population découle de plusieurs facteurs comme la stabilisation de la

RECHERCHES QUALITATIVES - Vol. 31(1), pp. 225-247.

RECHERCHE QUALITATIVE EN CONTEXTE AFRICAIN

ISSN 1715-8702 - http://www.recherche-qualitative.qc.ca/Revue.html

(C) 2012 Association pour la recherche qualitative 
fertilité et l'augmentation de l'espérance de vie. Il est à prévoir que le vieillissement s'accompagnera d'importantes répercussions sur toutes les sphères de la vie en général, mais sur les systèmes de santé en particulier (HelpAge International, 2000; ONU, 2002). Dès à présent, une réorganisation du système de santé s’impose au Sénégal afin de répondre de manière efficace et adaptée à la demande de soins et de services de la population vieillissante, notamment des femmes.

\section{Problématique}

En Afrique subsaharienne, les femmes représentent la majorité des personnes âgées de 60 ans et plus (ONU, 2002). Au Sénégal, notamment en milieu urbain, elles forment $52 \%$ de l'ensemble des personnes âgées (Ministère de l'Économie et des Finances (MEF), 2004). Comme l'ensemble des anciens du continent africain, ces femmes constituent un pilier sur lequel reposent la stabilité et la sécurité familiales, compte tenu de leur sagesse et de leur maîtrise des connaissances ancestrales (Tall, 2002). Pourtant, le poids du nombre et leurs responsabilités dans la gestion de la famille ne constituent pas un frein à leur discrimination. Les femmes sénégalaises vieillissantes font face, plus que leurs vis-à-vis masculins du même âge, à des conditions d'existence précaires et à des défis difficiles à relever pour assurer leur subsistance. En majorité, elles vieillissent sans conjoint parce que $42,6 \%$ d'entre elles sont des veuves contre 4,8 \% de veufs chez les hommes âgés (Coumé, Djiba, \& Ndiaye, 2000). Par ailleurs, 23,3\% de ces femmes sont dans un ménage de type polygamique (Signaté, 2006). Ce régime matrimonial constitue un facteur de déstabilisation à cause des conflits liés à la rivalité entre coépouses (MBow, 1996). En outre, très peu d'attention est accordée à certains de leurs problèmes de santé et aux perturbations psychosociales reliées au vieillissement tels la ménopause et le veuvage. Or, la pauvreté les oblige à négliger leurs soins de santé au profit de la satisfaction des besoins alimentaires de base de leurs familles (Couderc, 2004). Ces conditions ont des effets négatifs sur la santé des femmes et sur la signification qu'elles attribuent au vieillissement. Cet important défi peut nécessiter la mise en place de conditions particulières pour que les femmes vieillissent dans la dignité, c'est-à-dire qu'elles puissent vivre un grand âge associé à une sensation de satisfaction (Huang, 2003; Petry, 2003). Ainsi, le fait de saisir le sens de leurs expériences de vie constitue l'une des pierres angulaires à l'élaboration d'un programme de recherche et à l'application de mesures qui visent à leur offrir des services sensés et acceptables qui améliorent leurs conditions de santé et de bien-être.

Les écrits disponibles sur les expériences du vieillissement féminin sont encore rares et concernent exclusivement les pays asiatiques, européens et 
nord-américains. Ils démontrent la complexité du vieillissement qui est perçu comme un événement naturel, inévitable et individuel. Différentes expériences positives et négatives se dégagent de la recension des écrits.

\section{Les expériences positives}

Au-delà de 60 ans, le vieillissement féminin est un phénomène positivement vécu qui donne un sentiment de grande satisfaction et la possibilité de se réaliser au cours de la vie. Ainsi, des femmes ont indiqué que ce passage de la vie s'accompagne de sagesse et de cumul de connaissances qui incitent à un engagement au sein de la famille et de la communauté (DeVore, 1995; Durham, 2003; Petry, 2003; Sharon, 2000). En retour, elles se sentent respectées et en sécurité, tout en étant nourries par l'affection qui leur est manifestée (Huang, 2003). Le vieillissement est aussi un passage pendant lequel les femmes ont la perception d'être libres et plus autonomes dans leurs prises de décisions. Ce sentiment de liberté existentielle, engendrée par le départ des enfants de la maison familiale, est un facteur d'épanouissement pendant le grand âge (Huang, 2003; Petry, 2003). De façon paradoxale, Durham (2003) insiste sur le renforcement du réseau sociofamilial qui agit de façon positive sur l'expérience du vieillissement.

\section{Les expériences négatives}

Analysé de façon plus négative, le vieillissement favorise des changements physiques importants et une perte progressive de l'état de santé. Cette vulnérabilité provoque une perte de la confiance en soi et une réflexion continue orientée soit vers la vie soit vers la mort (Petry, 2003). Elle favorise la marginalisation des femmes vieillissantes par les jeunes qui les considèrent comme des fardeaux. Selon Dacher (1997), cet isolement est plus difficilement vécu lorsque le vieillissement se déroule dans un contexte d'incapacité fonctionnelle. Ainsi, dans son étude, l'importance de considérer plus humainement les femmes en situation d'incapacité physique a été suggérée dans le but de faciliter leur processus de vieillissement. La complexité du grand âge, chez des aînées à faible revenu, est également mise en exergue. En plus de l'angoisse liée à l'insécurité financière, le vieillissement se déroule très souvent dans un espace de vie très restreint et dans une solitude liée à l'effritement du réseau familial et social (Brunell \& Harrington, 2001). Une augmentation des besoins et des demandes de services sociaux et de santé est aussi largement évoquée comme étant une expérience du vieil âge féminin qui crée une forte dépendance à la communauté (Durham, 2003; Petry, 2003). Sous l'angle de l'influence, le vieillissement dépend des caractéristiques individuelles de nature biologique et de l'environnement culturel dans lequel vit la personne (Huang, 2003). Le développement de programmes facilite l'amélioration des 
interventions et des services communautaires offerts aux personnes vieillissantes.

Précisons qu'aucun écrit sur les expériences du vieil âge des femmes ne concerne l’Afrique, encore moins le Sénégal. Mais, les expériences positives recensées pourraient orienter la compréhension du vieillissement féminin dans ce continent, puisque la personne âgée jouit d'un statut honorable.

Cela contribue à la justification de la présente étude qualitative qui vise à comprendre la signification du vieillissement, selon la perspective de femmes sénégalaises âgées de 60 ans et plus vivant dans la communauté.

\section{Méthode de recherche}

\section{Approche de recherche}

À la lumière du but de la recherche, l'approche phénoménologique descriptive s'est imposée comme choix méthodologique afin de répondre de manière approfondie à la compréhension du sens que des femmes donnent aux expériences de vieillissement. La subjectivité de l'expérience humaine est mieux comprise par la phénoménologie (Giorgi, 1997; Van-Manen, 1990). Selon Deschamps (1993), la nature descriptive de l'expérience humaine telle qu'elle est vécue par la personne et à partir du point de vue de celle-ci fait partie des enjeux de la recherche phénoménologique.

La démarche de recherche phénoménologique de Giorgi (1997) a guidé la présente étude. Ce processus découle directement des principes eidétiques de Husserl (1970), ce qui nous éloigne des interprétations provenant des différentes ramifications de la phénoménologie husserlienne. Giorgi (1997) a cependant apporté quelques changements à la réflexion husserlienne afin de rendre pratique l'analyse des expériences vécues. À partir de la description de l'expérience humaine, Giorgi a mis en évidence un processus exclusivement centré sur l'analyse des données. Celle-ci est fondée sur l'utilisation d'unités de signification définies comme «les constituants qui déterminent le contexte du phénomène exploré et qui incluent forcément la part de la signification inhérente à ce contexte » (Deschamps, 1993, p. 18). Ainsi, Giorgi accorde une importance capitale à la description détaillée de l'ensemble des expériences telles qu'elles sont vécues, à la pratique de la réduction phénoménologique par le chercheur, à la recherche des essences, à l'exploitation des données brutes dans le langage de la discipline du chercheur et à la synthèse des résultats.

Inspirée des grandes étapes de toute recherche qualitative, la démarche de Giorgi $(1985,1997)$ comporte cinq étapes: 1) la collecte des données verbales; 2) la lecture des données; 3) la division des données en unités; 4) l'organisation et l'expression des données brutes dans le langage de la 
discipline, et 5) la synthèse des résultats. Ces étapes seront explicitées dans la section portant sur l'analyse des données.

\section{Le milieu de recherche et le choix des participantes}

L'étude s'est déroulée à Ouakam, un village traditionnel situé dans la banlieue ouest de Dakar. Les habitants de cette localité appartiennent à la communauté lébou. Ils constituent un groupe ethnique de pêcheurs, et font partie des premiers habitants de la région de Dakar anciennement dénommée la presqu'île du Cap-Vert. Le médecin-chef et un membre du comité de santé du centre de santé de la zone ont facilité le recrutement des participantes.

Pour participer à l'étude, les femmes âgées de 60 ans et plus devaient s'exprimer en langue nationale wolof et habiter le village traditionnel de Ouakam. Elles devaient être en mesure de mener des activités de la vie quotidienne, ce qui peut constituer un signe de bonne santé apparente.

Dix femmes âgées de 61 à 85 ans, avec un âge moyen de 75,7 ans, ont accepté de participer aux entrevues. De religion musulmane, ces femmes sont ménagères et en majorité des veuves ayant vécu une expérience de polygamie. Elles ont entre cinq et dix enfants âgés de 18 à 64 ans et habitent dans de grandes maisons multigénérationnelles partagées entre cinq et neuf ménages. Selon la classification de Duggleby, Bateman, et Singer (2002), quatre d'entre elles sont des «jeunes-vieilles" âgées de 61 à 71 ans, cinq sont des «vieilles-vieilles» de la tranche d'âge de 75 à 84 ans, et une femme " plus-vieille-vieille » est âgée de 85 ans.

\section{Déroulement de la collecte des données}

Les entrevues se sont déroulées au domicile des participantes. Elles ont été conduites par la chercheuse sur une période de 37 jours. Avant chaque entretien, une première visite de contact a été organisée afin d'expliquer à la participante les objectifs de l'étude et d'obtenir son consentement verbal. Les femmes ont par la suite été interviewées, de façon non structurée, à partir de l'une ou l'autre des deux questions suivantes : 1) racontez-moi, dans les détails, votre expérience du vieillissement telle que vous la vivez en ce moment; 2) que signifie pour vous le fait d'être une femme âgée de 60 ans et plus? Une incitation à la poursuite de la description des expériences vécues a été encouragée, lorsque les femmes observaient des temps d'arrêt dans la restitution de leur histoire. Avec leur autorisation verbale, les entrevues ont été enregistrées à l'aide d'un magnétophone numérique afin de faciliter, par la suite, la transcription des verbatims grâce au téléchargement direct des fichiers audio que permet l'appareil. Des notes manuscrites ont également été prises, par la chercheuse, tout au long de la collecte des données pour prévenir la perte d'informations en cas de défaillance du magnétophone. La durée moyenne des 
entrevues, déterminée en fonction de la disponibilité des participantes, a été d'une heure. De plus, à la fin de chacune des rencontres, un questionnaire sociodémographique a été rempli afin de recueillir le profil de chaque femme.

\section{Démarche d'analyse des données}

La recherche phénoménologique favorise la mise en forme des textes narratifs (Loiselle, Profetto-McGrath, Polit, \& Beck, 2007). Cette procédure a été suivie avec une grande minutie afin de respecter le plus fidèlement possible la transcription des expériences partagées par les femmes. Pour éviter la perte de signification, les verbatims ont été retranscrits et traduits mot à mot du wolof au français par la chercheuse qui parle couramment les deux langues. Certaines expressions wolof communément liées au vieillissement ont également été maintenues dans le texte et mises entre parenthèses. Le recours à l'exercice de la réduction phénoménologique a été maintenu tout le long du processus d'analyse des données.

En dehors de la collecte des données verbales, les quatre autres phases du processus d'analyse phénoménologique de données (Giorgi, 1997) ont été respectées :

- La lecture des données pour mieux appréhender l’idée générale de la description du phénomène, par la réécoute des entrevues sonores, la lecture répétée des verbatims et l'annotation des idées pertinentes qui s'imposaient à la lecture et qui, plus tard, ont facilité l'identification des thèmes et des sous-thèmes.

- La division des données en unités de signification, à partir d'une lecture très lente, a mené à la subdivision du récit en parties de textes qui correspondent à des faits relatifs au phénomène du vieillissement.

- L'organisation et l'expression des données brutes dans le langage de la discipline, pour comprendre le phénomène et approfondir les unités de signification. Avec des termes souvent utilisés en santé communautaire et le recours à la variation libre et imaginaire, les idées maîtresses contenues dans les extraits d'unités de signification ont été regroupées en sous-thèmes et en thèmes.

- La synthèse de l'ensemble des résultats qui a résumé les thèmes émergents en une seule structure générale représentant l'essence de l'expérience du vieillissement de femmes sénégalaises.

\section{Résultats}

À la suite de ces quatre étapes, les différentes synthèses et associations ont donné huit formes d'expériences de vieillissement des femmes sénégalaises rencontrées. Celles-ci renvoient à une composante (la structure générale). 


\section{Se sentir privilégiée d'avoir le statut de femme vieillissante}

Les femmes rencontrées considèrent que le vieillissement correspond à une étape d'épanouissement qui leur donne un statut, avec des avantages familiaux et communautaires considérables. Pour elles, les enfants représentent une richesse inestimable, une forme d'investissement familial et d'engagement dans le développement de la société. Après 60 ans, le fait d'avoir de grands enfants est perçu comme une "grâce de Dieu ». Ainsi, ces aînées évoquent la réussite de leurs enfants, la contribution financière de ces derniers aux charges de la maison, la sécurité qu'ils assurent pour leurs mères et l'affranchissement de celles-ci des travaux domestiques physiquement exigeants qui transforment l'étape de vieillesse en une période de grand repos.

L'appréciation de l'attention et du respect des membres de leur famille et de leur communauté constitue alors une expérience positive, chez ces femmes. Dans la société traditionnelle wolof, ces marques sont des valeurs culturelles et sociales importantes qui se perpétuent, malgré les mutations sociales négatives qui traversent la société contemporaine sénégalaise. Cet honneur allège d'une certaine manière le fardeau des pertes fonctionnelles résultant du grand âge et découle de plusieurs facteurs dont le plus important est le respect de la hiérarchie sociale.

Ce n'est pas trop une chance d'être respectée par les plus jeunes, c’est comme ça que fonctionne la communauté lébou [...] Ici, c'est le respect de la hiérarchie sociale et aucun jeune ne doit critiquer une personne plus âgée que lui (Marème).

Les femmes ont également beaucoup discuté de l'influence de l'âge sur leur tenue vestimentaire. Elles expliquent qu'elles choisissent leurs vêtements pour répondre non plus à des critères de séduction, mais davantage à un besoin de réalisation de soi, de fierté, d'épanouissement, de confort et de respectabilité. L'adoption d'un style vestimentaire particulier est aussi influencée par les croyances religieuses. Puisque leur foi islamique s'exprime de façon plus accentuée avec l'âge, ces femmes ont une tendance à couvrir tout leur corps. Le port d'une tenue typique fait alors foi de dignité, de maturité et de sagesse. Les femmes sénégalaises rencontrées disent se vêtir selon les convenances, avec des "vêtements de femmes vieillissantes », essentiellement des robes longues et de grands boubous simples, amples, amidonnés et repassés, avec un mouchoir de tête et un grand voile par-dessus le corps.

Ce sont ces habits qui donnent de la prestance à la personne vieillissante. Je ne peux pas porter certaines tenues [...] Je ne parle même pas de mon état de femme vieillissante ni du statut de mon mari dans le village, mais du respect que la société doit à mes 
enfants à travers mon code vestimentaire, mes paroles et mes comportements (Amsatou).

\section{Adopter de nouveaux rôles valorisés par la famille et la communauté}

Les participantes disent s'engager dans de nouvelles fonctions sociofamiliales compatibles avec leur grand âge. D'abord, elles mettent l'accent sur leur rôle de grand-mère dont les responsabilités constituent un motif de fierté pour toute femme d’âge mûr. La majorité de ces femmes sénégalaises voient même en leurs petits-enfants une raison de vivre. « Je peux même dire que le fait d'avoir des petits-enfants est pour moi un cadeau du ciel. Je me demande si j'aurais pu survivre sans des petits-enfants » (Astou). L'exercice de ce rôle signifie le maintien avec fierté d'une complicité entre les femmes et leurs petits descendants. Cette relation est parfois plus étroite que celle qu'elles ont avec leurs propres enfants. Leur implication se situe dans l'éducation traditionnelle et religieuse, les soins et la surveillance à domicile de leurs jeunes petits-enfants.

Ensuite, les participantes mentionnent le leadership qu'elles prennent dans les cérémonies familiales. Cette expérience est particulièrement valorisante, dans le vieil âge féminin. Au Sénégal, bien que les hommes soient traditionnellement les chefs et les décideurs au sein des familles, ils ne sont pas pour autant engagés dans l'organisation des rassemblements comme les baptêmes, les mariages, les funérailles et les grandes réunions familiales. Ces événements très importants dans le contexte sénégalais sont en grande partie dirigés par les femmes. Puisque l'âge détermine la position de porte-parole dans les familles, pour ce genre d'événements, les femmes qui sont les plus âgées accomplissent les responsabilités qui découlent de la gestion et de la coordination de ces rencontres festives. Ce rôle est ainsi un privilège qui donne aux participantes le sentiment de rester encore utiles, en renforçant la cohésion familiale. D’une certaine manière, ceci a une influence positive considérable sur leur épanouissement.

Moi, dans ma famille, je suis l'aînée et je joue un rôle pivot. Lorsqu'il y a une cérémonie, je passe au moins trois jours avec les membres de ma famille pour superviser les préparatifs (Astou).

Enfin, la majorité des femmes rencontrées soulignent leur rôle de conseillère matrimoniale. À titre de gardiennes des valeurs culturelles et traditionnelles, les participantes trouvent important d'offrir une assistance aux jeunes mariées qui, avec la modernité, divorcent d'autant plus facilement. Cette forme d'implication sociale est ainsi perçue par certaines comme la garantie de la stabilité des ménages, le divorce ne pouvant survenir que d'une façon très exceptionnelle. 
Si le mariage trébuche, nous (les aînées) sommes là pour le soutenir, pour accompagner le couple jusqu'à ce que les liens du mariage soient solidement maintenus (Mbissine).

Les participantes disent alors posséder des qualités humaines pour s'acquitter de ce rôle qui contribue au maintien de l'unité sociofamiliale. Elles se sentent encore très utiles lorsqu'elles se réfèrent à leur expérience pour accompagner les jeunes femmes dans leur ménage.

Je suis très sage, sincère et discrète. Je ne rapporte jamais les secrets que les femmes ou leurs conjoints me confient. Cela peut engendrer des conflits et même un divorce. Or, je ne dois pas être responsable de telles bévues (Amsatou).

\section{Être confrontée à une vulnérabilité physique}

Comme le reconnaissent toutes les femmes rencontrées, être vulnérable physiquement fait partie intégrante du processus du vieillissement humain. Cette vulnérabilité se définit comme la fragilité et l'affaiblissement tant du fonctionnement des organes musculo-squelettiques et sensoriels que de l'état de santé général. Les participantes mentionnent que, pour elles, les signes de vulnérabilité physique débutent vers l'âge de 50 ans et augmentent progressivement en intensité avec les années. Elles déclarent faire appel à différentes stratégies d'adaptation pour apprivoiser cet état de fragilité et pour bien vieillir. Ainsi, les femmes rencontrées disent prendre conscience graduellement de la perte de leurs capacités fonctionnelles, apprendre à vivre avec une détérioration progressive de leur état de santé. De fait, «lorsqu’on demande à Dieu une longue vie, il faut aussi s'attendre à ce que cette longue vie s'accompagne de déficiences de toutes sortes » (Dieyna). Par conséquent, les multiples formes de changements vécus avec l'âge qui avance font partie des expériences du vieillissement des femmes rencontrées. Qu'il s'agisse de la baisse de capacités physiques ou de changements corporels externes, ces transformations sont avant tout ressenties par les femmes comme le déclin naturel et personnel des fonctions de certains organes. Ce sont des événements généralement bien acceptés pour la plupart d’entre elles.

Au fond, je ne me plains pas de mes changements corporels parce que j'ai toujours souhaité avoir une longue vie. C'est pourquoi je remercie Dieu de m'avoir donné ces transformations qui accompagnent forcément la vieillesse et que j'accepte avec satisfaction (Mbissine).

Ces femmes adoptent ainsi de meilleures habitudes de vie comme le maintien d'un mode de vie actif, le suivi de leur état de santé et l'amélioration 
de leur alimentation, pour prévenir l'aggravation de leurs douleurs articulaires et retarder l'évolution des affections liées au vieillissement.

\section{Rencontrer des obstacles dans l'accès aux soins de santé}

Les obstacles dans l'accès aux soins de santé constituent un thème central de notre étude dans les expériences vécues du vieillissement. Compte tenu de la diminution des capacités fonctionnelles et de la détérioration progressive de leur état de santé, les femmes perçoivent la fréquentation des services de santé comme une priorité. Cependant, de nombreux obstacles se dressent devant leurs tentatives d'accéder à des soins de qualité.

Les participantes ont discuté de nombreuses barrières financières qui influent sur leur accès aux services de santé. Ces obstacles sont liés aux difficultés de prise en charge médicale. Pour plusieurs, l'accès facile à la consultation médicale et aux médicaments prescrits par un médecin est un privilège réservé uniquement à une minorité de femmes vieillissantes. De fait, l'impossibilité de payer les coûts de tels services est constamment apparue dans les entrevues, obligeant l'ensemble des participantes à renoncer malgré elles à la fréquentation des services de santé. Selon ces participantes, cette épineuse question a des causes multiples qui relèvent essentiellement de l'absence d'assurance maladie et de revenus des personnes vieillissantes. Pour plusieurs, l'application de la politique de gratuité des soins accordée aux personnes âgées de 65 ans et plus ne semble pas les concerner. Elles disent continuer de payer des services auxquels elles auraient droit gratuitement. De plus, certaines femmes disent ne pas respecter le suivi de leurs traitements et la surveillance de leurs maladies parce qu'elles ne disposent pas de ressources financières suffisantes.

Le prix de la consultation médicale et celui des médicaments, il faut les prévoir. Si j'embarque dans ce processus, je veux dire aller au centre de santé, je dois le faire assez souvent parce que j'ai des maladies chroniques. Or, je n'en ai pas les moyens (Dieyna).

D'autres rendent compte de l'insatisfaction dans les soins découlant de l'insuffisance et de l'incompétence du personnel de santé. En effet, elles soulignent avoir consulté plusieurs spécialistes sans vraiment trouver la cause de leur affection, encore moins obtenir des médicaments efficaces pour leur état de santé. La longue attente qui précède l'offre de soins de santé a aussi été perçue comme une source d'inconfort que plusieurs disent ne pas être disposées à endurer pour bénéficier de services de santé professionnels. Cette situation suscite plus de doute sur leur santé que de bien et oblige les participantes à considérer l'accès aux soins de santé comme « un parcours du combattant » (Sagar). 


\section{Faire face à une grande vulnérabilité psychologique}

Les femmes ont insisté sur leur vulnérabilité psychologique qu'elles décrivent comme un événement de fin du parcours de vie. Selon elles, cette problématique provient de leurs expériences antérieures qui influencent leurs comportements, pour le reste de leur vie.

Cette vulnérabilité concerne tout d'abord l'acceptation de la mort. Bien que peu souhaitée, la mort est perçue comme un malheur, un phénomène très personnel et normal, un destin auquel aucune personne ne peut échapper parce que "toute chose a une fin" (Astou). Les participantes se font ainsi une conception de cette expérience dont le simple fait d'y penser est une façon de croire en Dieu. Elles s'y en attendent, l'acceptent et se préparent à l'affronter avec sérénité.

Je n'ai pas peur de la mort [...] Je peux dire que mes 84 ans constituent un privilège pour moi. Si je mourais aujourd'hui, je serais bien dans ma tombe (Ngagne).

La grande fragilité psychologique englobe aussi les deuils successivement vécus dans la famille. Pour les femmes sénégalaises de l'étude, ces expériences très bouleversantes engendrent beaucoup de tristesse, à l'étape de vieillissement où le rêve de vivre encore plus longtemps n'est plus permis. Ainsi, pour ces femmes âgées de 60 ans et plus, l'annonce d'un décès suscite souvent une réflexion sur la vie et une interrogation sur leur propre mort. Elles sont convaincues que les décès fréquents et successifs des membres de leur entourage familial sont des indices majeurs de l'approche de leur propre mort.

J'ai perdu mon mari, mon frère qui n'avait que 41 ans et presque tous les hommes et les femmes de mon âge. Mes deux parents sont décédés et ma fille que j’ai mise au monde. Ces pertes à répétition signifient que moi aussi je vais bientôt quitter ce monde (Marème).

Le renforcement des liens avec les amies du même âge présente également beaucoup d'aspects émotifs chez les participantes. Celles-ci ont plusieurs fois abordé la nécessité d’atténuer la solitude qui leur donne l'impression d'être malades et abandonnées, les émotions émanant de la perte d'êtres chers et la réalité d'une fin de vie qui approche.

Les amies constituent une des possibilités les plus efficaces pour continuer de donner un sens à leur vieillissement, pour trouver du réconfort et de la compagnie, pour rire et pour se donner du plaisir. Ces liens affectueux s'appuient sur le partage d'activités religieuses ou récréatives, de joies, de difficultés de la vie et de problèmes rencontrés par les enfants qui grandissent. 
« Les amies, c'est beaucoup trop important parce que nous sommes des veuves et les enfants sont presque tous partis vivre chez eux » (Fatou).

La majorité des femmes rencontrées affirment avoir vécu, en vieillissant, l'expérience d'aidante naturelle auprès de leur conjoint. Selon elles, tenir le rôle d'aidante naturelle est un devoir conjugal très noble qui permet de suppléer les besoins d'un conjoint malade, pendant une période de deux à dix ans. Les aidantes naturelles rencontrées rapportent qu'elles se sont acquittées de leur devoir d'assistance de façon remarquable, en faisant preuve de discrétion, de disponibilité, de compassion et d'abnégation. Ce rôle est tout de même un événement douloureux qui s’inscrit désormais comme une partie intégrante de l'existence des femmes vieillissantes rencontrées, avec des conséquences d'ordre sanitaire et psychologique qui perdurent bien après le décès du partenaire.

Dix ans pendant lesquels je n'avais plus de vie personnelle, ma vie dépendait entièrement de celle de mon mari [...] J'avais fait le possible et l'impossible et j'étais fatiguée, presque déprimée. C'est après son décès que j’ai commencé à souffrir de toutes sortes de problèmes de santé (Fatou).

\section{Faire face à des expériences de vulnérabilité économique}

Malgré tout le respect et l'attention que l'entourage accorde aux femmes vieillissantes, plusieurs vivent une situation de vulnérabilité économique qui est perçue comme une expérience intimement associée à leur fin de vie. Pour certaines, cette situation correspond à une absence totale de ressources financières ou à une insuffisance de revenus pour satisfaire leurs besoins les plus élémentaires comme nourrir et habiller la famille, payer les soins de santé et les factures d'eau et d'électricité. Divers facteurs contribuent à cette situation de vulnérabilité économique. Le plus important est que plusieurs femmes rencontrées sont devenues, en vieillissant, des femmes-chefs de ménage. Cette responsabilité qui les oblige à prendre en charge une partie ou la totalité des dépenses du foyer est moralement et matériellement une source de fatigue et de désespoir. Elle les oblige à développer et à mettre en œuvre des stratégies de survie familiale comme le recours au petit commerce et à la solidarité familiale. Ces femmes sénégalaises sont devenues en vieillissant de grandes « débrouillardes » $(\text { gorgorlu })^{1}$. Selon elles, se débrouiller consiste à réfléchir le soir à des solutions au point d'en perdre le sommeil, et à se lever très tôt le matin pour étudier les moyens permettant d'obtenir des denrées alimentaires pour le repas familial de la journée.

Si je ne trouve pas la dépense quotidienne, on ne fait pas la cuisine dans la maison. Mais, je peux toujours demander à un membre de 
ma famille élargie de nous donner à manger, même si la quantité n'est jamais suffisante [...] il faut remplir le ventre de mes petits-enfants. Le reste comme le lait, les fruits et des choses comme ça relève du luxe (Yakhara).

\section{S'adapter à un mode de vie qui oscille entre tradition et modernité}

Les femmes sénégalaises de l'étude disent demeurer dans deux contextes de vie partagés entre un présent moderne et un passé traditionnel. Cette situation génère différentes expériences parfois très contradictoires qui influent sur leur fin de vie. Les femmes rencontrées reconnaissent les privilèges que leur offre la vie moderne. Elles sont d'accord que les conditions de vie du passé se sont améliorées, avec les progrès scientifiques. "Tout ce qui était difficile hier est devenu plus facile maintenant» (Mbissine). Ainsi, faisant un constat de différence entre l'époque de leur enfance et même de leur âge adulte et aujourd'hui, elles se sentent très émerveillées par la modernité avec ses conditions de vie facile et agréable du fait des progrès du siècle présent. Elles évoquent pour les filles l'accès à l'école, au travail rémunéré, aux voyages et loisirs et aux nouvelles technologies qui améliorent l'information et allègent les tâches ménagères.

Les femmes modernes ne connaissent pas les travaux domestiques pénibles puisqu'elles ont des machines pour préparer le mil, du gaz pour faire la cuisine et des domestiques qui travaillent à leur place (Fatou).

À l'inverse, les participantes expriment des ennuis quant aux changements de valeurs socioculturelles. Elles considèrent que la modernité a apporté plusieurs changements dans leur vie. Compte tenu de leur éducation traditionnelle différente de celle que connaissent les jeunes, elles disent s'adapter difficilement à la vie moderne dont elles ont une perception assez négative. Selon elles, la modernité est plutôt source d'inconforts pour des femmes vieillissantes en termes de dérive de l'éducation traditionnelle des jeunes. Elles dénoncent l'influence négative de la télévision sur le comportement des jeunes et la transformation de la société qui devient de plus en plus artificielle et matérialiste avec comme corolaire la valorisation du désir de bien paraître.

Maintenant, c'est plutôt le paraître qui donne une notoriété et, non plus, le titre de noblesse familiale qui était très respecté dans le passé [...] Je ne me sens pas à l'aise avec la modernité, car je ne suis pas ce que je devais être, je ne suis pas non plus dans le monde que je considère comme étant le contexte de vie d'une femme vieillissante (Ngagne). 


\section{Accorder une importance prioritaire à la religion}

Une autre expérience renvoie à la dimension spirituelle du vieillissement. Il s'agit des expériences en lien avec la religion et auxquelles les participantes attachent une importance toute particulière. Selon le discours des femmes sénégalaises rencontrées, le grand âge constitue un moment de rapprochement à Dieu. De leur avis, le culte religieux est un ensemble de connaissances et d'activités qui permet d'être en communion avec leur Créateur et d'en tirer une satisfaction morale et une sérénité qui accompagnent leur fin de vie. Ces femmes affirment qu'il existe une nette différence dans la perception de l'exercice religieux avant et pendant l'étape de vieillesse. Dans leur jeunesse, elles ne se souciaient pas du respect rigoureux des principes de l'islam et maintenant, elles se sentent obligées de se conformer au rituel sacré de l'islam. D'ailleurs, la pratique de la religion constitue une forme de réalisation de soi et, en même temps, une sorte de refuge sécurisant qui favorise la tranquillité d'esprit et entretient l'espoir quant à l'avenir. Le fait de vivre 60 ans et plus est considéré comme une faveur divine qui mérite de rendre grâce à Dieu. Ainsi, avec le temps qui passe, la pratique religieuse revêt une signification toute particulière et est devenue systématique chez les femmes sénégalaises d'âge avancé. En premier lieu, celles-ci parlent du renforcement de leurs croyances religieuses. En second lieu, elles disent se conformer aux préceptes de l'islam par l'accomplissement systématique des cinq piliers de l'islam, surtout la prière ( $2^{\mathrm{e}}$ pilier) et le pèlerinage aux lieux saints de l'islam ( $5^{\mathrm{e}}$ pilier).

Je ne suis jamais tranquille lorsque je manque une heure de prière. C'est en invoquant les noms de Dieu que j'arrive à traverser les moments de solitude, lorsque mes amies ne sont pas là. Et jusqu'à présent, chaque fois que le pèlerinage approche, je ressens un désir ardent de retourner à La Mecque (Fatou).

De plus, plusieurs participantes ont vécu l'expérience du veuvage qui est assujettie au respect strict de devoirs religieux. Ainsi, les veuves rencontrées mentionnent qu'elles ont assimilé beaucoup de connaissances sur leur religion, particulièrement lors des premiers jours de leur veuvage. Il s'agit d'un savoir qu'elles continuent d'utiliser pour renforcer leur exercice religieux, et ça, pour tout le temps qui leur reste à vivre. Tout en affirmant avoir fait beaucoup de prières pour le repos de l'âme de leur conjoint, elles soulignent que cette étape marquée par la méditation est très influencée par l'environnement socioculturel local. Comme attestent certaines, les contraintes traditionnelles rendent cette expérience particulièrement difficile à vivre puisque le veuvage la transforme en une période de privations assorties de perceptions et de représentations. Dans la coutume wolof, une veuve doit être très sobrement habillée, elle doit 
rester discrète dans ses manières de faire qui sont souvent en contradiction avec les comportements adoptés habituellement.

J'ai passé 130 jours à réciter le Coran et à prononcer Astahfirulah $^{2}$. Je sortais de temps en temps, seulement pendant la nuit, pour éviter les regards, pour éviter aussi de rencontrer des gens qui ne souhaitaient pas me voir avec mes habits de couleur sombre (Astou).

\section{La structure générale du vieillissement}

De l'analyse des données de l'ensemble des thèmes ressort une structure générale de l'expérience du vieillissement de femmes sénégalaises âgées de 60 ans et plus. Les dix participantes avouent vivre un paradoxe : le vieillissement correspond à une expérience d'épanouissement malgré une vulnérabilité physique, psychologique et économique amenant une réflexion existentielle.

\section{Discussion des résultats}

Les résultats de la présente étude corroborent plusieurs constats faits dans d'autres études portant sur la signification du vieillissement.

D’une part, ce processus permet aux femmes de bénéficier de privilèges qui favorisent leur épanouissement en lien avec des marques de respect et d'attention que leur témoignent la famille et la communauté. De plus, le vieillissement encourage, chez les participantes, l'adoption d'un mode de vie et d'une attitude qui augmentent leur respectabilité par les membres de leur entourage. En outre, les femmes rencontrées démontrent leur utilité sociale, à travers différents rôles qu'elles s'approprient, dans le cadre du renforcement des liens amicaux, familiaux et communautaires. Ces expériences profondément ancrées dans les valeurs partagées par les sociétés africaines constituent, chez les femmes, la récompense pour les efforts consentis tout au long de la vie, comme conjointes et comme mères exemplaires. L'information sur cette forme d'expérience heureuse du vieillissement corrobore et complète aussi les résultats d'autres chercheurs (Caldas \& Bertérö, 2007; Jonas, 1992; Namakando-Phiri, 2004) qui soutiennent que le nouveau mode de vie est fortement influencé par l'occident. Toutefois, dans plusieurs pays qui partagent un investissement socioculturel important, les égards envers la famille et la communauté et le respect de la séniorité demeurent une réalité. Ainsi, les aînés sont d'autant plus choyés qu'ils sont nourris, soignés et habillés par leur famille (Jonas, 1992; Namakando-Phiri, 2004). Ces résultats convergent aussi avec ceux de Bopp (1996) qui souligne que, dans le Sénégal traditionnel, les femmes doivent une obéissance permanente à leurs parents, puis à leur conjoint et à leur belle-famille, pour assurer leur avenir personnel et pour asseoir la notoriété de leurs enfants. C'est seulement lorsqu'elles vieillissent et qu'elles ont des 
enfants adultes chefs de famille qu'elles peuvent se libérer de cette double autorité. À leur bonheur, elles changent de statut et deviennent des femmes plus respectées et choyées par leur entourage.

L'étude démontre que le fait de prendre de l'âge expose les femmes sénégalaises à diverses sources de vulnérabilité. Celles-ci vivent une détérioration graduelle de leur état de santé, à l'instar d'autres femmes vieillissantes comme le soulignent les résultats de plusieurs recherches (DeVore, 1995; Durham, 2003; Namakando-Phiri, 2004; Sharon, 2000; Shin, Kim, \& Kim, 2003). Ces conclusions rejoignent également celles de Signaté (2006) qui a déjà observé, chez des aînés sénégalais, certaines maladies liées à la mauvaise alimentation comme l'anémie, le diabète de type II, l'hypertension et des troubles de la vue. Soucieuses de la détérioration de leurs capacités fonctionnelles, les femmes de l'étude adoptent alors des comportements comme le maintien d'un mode de vie actif, le suivi de la santé et l'amélioration de leur alimentation. Ces activités de prévention ont été antérieurement révélées auprès de Camerounais vieillissants qui profitent des campagnes de sensibilisation et des consultations médicales organisées dans la communauté (Kuaté-Défo, 2005).

Nos résultats mentionnent que la perspective d'une meilleure qualité de vie amène les femmes de la présente étude à faire appel à différentes stratégies pour s'adapter aux modifications physiques reliées au grand âge. L'étude indique que les participantes adoptent un mode de vie actif afin de garder le corps et l'esprit alertes, prévenir l'aggravation des douleurs articulaires et ralentir l'évolution des affections liées au vieillissement. Ces comportements corroborent les résultats d'autres recherches où les personnes sollicitent fortement leur sens de l'imagination pour s'adapter au grand âge (Caldas \& Bertérö, 2007; Huang, 2003).

Alors que leur condition de santé requiert une prise en charge personnalisée, les femmes se heurtent à diverses barrières dans l'accès à des soins de santé dignes et de qualité. Le grand âge est ainsi perçu comme une période de la vie où les possibilités de soins signifiants sont rares. Ces conditions sont fréquemment vécues dans les pays du sud où les aînés rencontrent des difficultés pour supporter les coûts des consultations médicales et des médicaments (HelpAge International, 2002). Dans un tel contexte, ces femmes sénégalaises ont le sentiment d'être les "grandes oubliées » de la politique nationale de santé, malgré la gratuité des soins offerts aux personnes du $3^{\mathrm{e}}$ âge. Comme les répondants d'une étude zambienne, elles se tournent donc vers des approches traditionnelles de soins pour contrer les différents 
symptômes et pathologies qui se manifestent tout au long de leur vieillissement (Namakando-Phiri, 2004).

Pendant le grand âge, les participantes de l'étude disent éprouver des expériences de santé mentale très difficiles. Le vieillissement devient pour elles une traversée de deuils successifs et fréquents dans leur entourage immédiat. C'est à ce stade de l'existence que ces femmes prennent conscience que leur fin de vie approche. En outre, comme plusieurs autres aînés de leur âge, elles acceptent la mort comme un phénomène naturel, une issue inévitable remplie de mystères et d'inquiétudes, et en même temps d'espoir de la possibilité d'une « après-vie » heureuse (Cossette \& Pépin, 2001; Shin et al., 2003).

Vieillir, c'est également connaître la solitude que ces femmes tentent de combler par le maintien et par le renforcement de liens avec des amies de même âge. Auprès de celles-ci, elles trouvent sécurité, réconfort et compassion, surtout lors d'événements douloureux comme la maladie, la perte d'êtres chers et la solitude à la suite du départ des grands enfants du domicile parental. Ce résultat abonde en partie dans le même sens que la théorie de la sous-culture (Rose, 1965) qui explique la tendance des personnes âgées à se regrouper, selon leur vision de la vie, pour mieux interagir. Il rejoint également les conclusions d'études qui indiquent que l'univers amical initié ou nourri génère une intense satisfaction qui permet aux aînés d'apprendre des autres et de continuer à se réaliser (Caldas \& Bertérö, 2007; DeVore 1995; Namakando-Phiri, 2004; Petry, 2003). Il est probable que cette réalité soit à l'origine du proverbe wolof qui soutient que «l'homme est le remède de l'homme (nit nitay garabam) ». En d'autres termes, ce dicton signifie que les relations humaines constituent une ressource pour la survie. Elles améliorent le quotidien chez les personnes d'âge mûr.

Enfin, tout au long de l'expérience du vieillissement, les participantes avouent rencontrer des difficultés économiques qui s'accentuent au fur et à mesure que leur âge augmente. De fait, avec le veuvage qui accompagne fréquemment le processus de vieillir, elles deviennent, comme plusieurs Africaines, des femmes-chefs de ménage responsables d'une famille nombreuse, mais sans soutien financier (Darkwa \& Mazibuko, 2002; HelpAge International, 2002). Au cours du grand âge, elles sont encore obligées de rechercher des revenus pour satisfaire leurs besoins les plus élémentaires comme la nourriture et les soins de santé. Pour survivre, elles peuvent devenir des commerçantes de détail ou se trouvent obligées de recourir à la solidarité familiale et communautaire de plus en plus rare (Kane, 1996; Namakando-Phiri, 2004). 
Le vieillissement est aussi un processus de réflexion sur l'existence. Ces femmes se trouvent à devoir concilier leurs référents traditionnels dans un contexte de plus en plus moderne. Avec les tensions vécues entre le passé, le présent et l'avenir, il est intéressant de voir, à l'instar de ce qui est vécu ailleurs dans le monde, comment les femmes sénégalaises vieillissantes reconnaissent les progrès accomplis dans la condition de la femme. Tout en préservant leur identité, elles critiquent les pièges qu'elles voient dans ces avancées. Ainsi, pour ces femmes sénégalaises très humbles, la vieillesse est une étape de nostalgie des habitudes de vie passée et de certaines valeurs anciennes qui guidaient la vie, tout en favorisant l'harmonie et la solidarité entre les membres de la communauté. Comme dans certaines études, prendre de l'âge c'est donc faire l'expérience d'une vie qui présente des contradictions et des inconforts créés par les changements de valeurs sociales et culturelles, notamment en lien avec l'éducation des jeunes et la primauté de l'intérêt individuel sur celui de la communauté (Jonas, 1992; Namakando-Phiri, 2004). Cependant, la modernité, avec ses conditions matérielles auxquelles ont accès les jeunes générations, émerveille les femmes rencontrées, telles les conclusions de l'étude de Kane (1996).

Le vieillissement inclut également une dimension religieuse à laquelle ces femmes accordent une attention toute particulière. L'engagement religieux représente un critère très important de sagesse et de valorisation sociale. C'est au cours du $3^{\mathrm{e}}$ âge qu'elles disposent d'assez de temps pour se consacrer à leur rapprochement avec Dieu. Ces femmes sénégalaises profitent du temps que leur offre le vieillissement pour mieux connaître leur religion et pour davantage se conformer à la pratique assidue des cinq prières journalières accompagnées systématiquement de rituels telles les ablutions et la récitation de psaumes. L'âge mûr leur donne aussi l'occasion de fréquenter assidûment la grande mosquée et d'être présentes aux grandes cérémonies religieuses, vêtues fièrement d'habits décents de préférence blancs. C'est aussi en prenant de l'âge qu'elles aspirent davantage à une vie plus longue qui leur donne le temps et les moyens de réaliser des rêves tel l'accomplissement du pèlerinage à La Mecque (dernier pilier de l'islam). Ces résultats corroborent ceux de Namakando-Phiri (2004) qui soutient que, parmi les plus grandes valeurs partagées par les aînés, qui donnent sens à leur existence, on trouve le respect des principes religieux. La religion devient un refuge qui permet de supporter les épreuves incontournables du vieillissement, d'affronter la mort en toute sérénité et de s'attendre à une vie sans supplices après la mort. Il s'agit ici d'une perspective intéressante qui débouche sur une opportunité à développer entre la vie spirituelle et la santé. 


\section{Conclusion}

Notre étude a globalement facilité la connaissance approfondie du vieillissement. Ce processus dynamique est décrit, par des femmes sénégalaises âgées de 60 ans et plus et vivant dans la communauté, comme un ensemble d’expériences multiformes, vécues de façon assez contradictoire, qui comportent à la fois des satisfactions et des contraintes. La richesse des descriptions faites par des femmes sur leurs propres expériences témoigne de la complexité du vieillissement féminin. Ainsi, le sens du vieillissement est généralement celui d'une expérience riche décrite comme une plénitude vécue dans un contexte de vie traditionnelle typique qui tranche avec la représentation d'un phénomène de pertes, de deuils, de renoncements même si ceux-ci sont quand même présents.

En permettant cette compréhension du vieillissement, l'étude constitue une contribution originale qui peut servir de point de départ à de nouvelles recherches qui se feront désormais sur le grand âge féminin. Des recommandations sont ainsi apportées dans le sens de la mise en œuvre d'un programme de recherche visant l'amélioration des conditions de vie et de santé des femmes âgées de 60 ans et plus. Elles invitent de même à poursuivre la réflexion afin que d'autres préoccupations de femmes vieillissantes soient étudiées. Un tel programme de recherche est nécessaire pour disposer de connaissances plus approfondies et plus globales sur le vieillissement féminin.

Dans la mesure où, depuis 2006, le Sénégal s'est engagé dans l'amélioration des conditions de vie des aînés, les différentes contributions de cette étude sur le vieillissement s'inscrivent parfaitement dans les nouvelles préoccupations de ce pays.

\section{Notes}

1 Terme sénégalais utilisé pour désigner une personne qui fait des efforts ou qui se débrouille au quotidien, pour survivre ou pour faire vivre sa famille.

2 Expression prononcée lorsqu’on demande pardon à Dieu pour les pêchés commis consciemment et inconsciemment.

\section{Références}

Bopp, C. (1996). Les femmes-chefs de famille à Dakar. Dans J. Bissiliat (Éd.), Femmes du sud, chefs de famille (pp. 129-149). Paris : Éditions Karthala. 
Brunell, M., \& Harrington, C. C. (2001). Doing for others : it gives me something to do. An ethnographic study of older, urban women living alone in poverty (Thèse de doctorat inédite). Columbia University Teachers College, New York. Repéré à http://www.ecsocman.edu.ru/text/19297962/

Caldas, C. P., \& Bertérö, C. (2007). Living as an oldest old in Rio de Janeiro : the lived experience told. Nursing Science Quarterly, 2(4), 376-382.

Cossette, R., \& Pépin, J. (2001). Vieillir et croître à travers les déclins, un défi spirituel avant tout. Théologiques, 9(2), 47-67.

Couderc, M. (2004, Août). Les retraités sont fatigués. Communication présentée à la Conférence sur le vieillissement en Afrique, Johannesburg, Afrique du Sud.

Coumé, M., Djiba, B., \& Ndiaye, A.S. (2000). Étude sur les besoins sociosanitaires et économiques des personnes âgées au Sénégal (Rapport de recherche). Dakar, Sénégal : Ministère de la Famille, de l’Action sociale et de la Solidarité nationale, Direction de l'action sociale.

Dacher, J. E. (1997). Older women's narratives of aging, disability, and participation in a rehabilitation program : a phenomenological study of lived experience (Thèse de doctorat inédite). University of Albany, United States of America.

Darkwa, O. K., \& Mazibuko, F. N. M. (2002). Population aging and its impact on elderly welfare in Africa. The international journal of aging and human development, 54(2), 107-123.

Deschamps, C. (1993). L'approche phénoménologique en recherche: comprendre en retournant au vécu de l'expérience humaine. Montréal : Guérin.

DeVore, C. W. (1995). Life as experienced by women in their sixties : a phenomenological study (Thèse de doctorat inédite). University of Colorado, United States of America.

Duggleby, W., Bateman, J., \& Singer, S. (2002). The aging experience of well elderly women : initial results. Nursing \& Health Sciences, 4(3), A10.

Durham, C. (2003). An examination of the life experiences of three communitydwelling older adult women (Thèse de doctorat inédite). United States International University, San Diego, United States of America.

Giorgi, A. (1985). Phenomenology and psychological research. Pittsburgh : Duquesne University Press. 
Giorgi, A. (1997). De la méthode phénoménologique utilisée comme mode de recherche qualitative en sciences humaines : théorie, pratique et évaluation. Dans J. Poupart, J. P. Deslauriers, L. H. Groulx, A. R. Laperrière, R. Mayer, \& A.P. Pires (Éds), La recherche qualitative: enjeux épistémologiques et méthodologiques (pp. 341-364). Montréal: Gaëtan Morin.

HelpAge International. (2000). Information needs for research, policy and action on ageing and older adults : a report of the follow-up meeting to the 2000 Harare MDS workshop, indicators for the minimum data set project on ageing: a critical review in sub-Saharan Africa. Repéré à http://extranet.who.int/iris/handle/123456789/150?locale=fr

HelpAge International. (2002). State of the world's older people 2002. Repéré à http://www.abuel.org/docs/09_helpage.pdf

Huang, C. Y. (2003). Taiwanese elders : successful aging and physical activity. (Thèse de doctorat inédite). Universitry of Incarnate Word, San Antonio, United States of America.

Husserl, E. (1970). The crisis of European sciences and transcendental phenomenology: an introduction to phenomenological philosophy. Evanston: Northwestern University Press. (Ouvrage original publié en 1934-1937).

Jonas, C. M. (1992). The meaning of being an elder in Nepal. Nursing Science Quarterly, 5(4), 171-175.

Kane, M. (1996). Étude des stratégies de résolution de problèmes des personnes âgées retraitées de Dakar, en vue de l'élaboration, à leur bénéfice, d'une politique de protection sociale, dans une perspective de développement social (Thèse de doctorat inédite). Université Laval, Québec, Canada.

Kuaté-Défo, B. (2005). Facteurs associés à la santé perçue et à la capacité fonctionnelle des personnes âgées dans la préfecture de Bandjoun au Cameroun. Cahiers québécois de démographie, 34(1), 1-46.

Loiselle, C. G., Profetto-McGrath, J., Polit, D. F., \& Beck, C. T. (2007). Méthodes de recherche en sciences infirmières : approches quantitatives et qualitatives. Québec : Éditions du renouveau pédagogique.

MBow, P. (1996). Femmes, violence et religions. Revue trimestrielle de l'Institut africain pour la démocratie, 6, 75-82. 
Ministère de l'Économie et des Finances (MEF). (2004). La pauvreté au Sénégal : de la dévaluation de 1994 à 2001-2002 : version préliminaire. Dakar : Direction de la prévision et de la statistique.

Namakando-Phiri, N. A. (2004). A phenomenological study of ageing amongst the older persons in Zambia (Thèse de doctorat inédite). University of South Africa, Afrique du Sud.

Organisation des Nations Unies (ONU). (2002). World population ageing 1950-2050. New-York : United Nations publication.

Petry, H. (2003). Aging happens-experiences of Swiss women living alone. Journal of Women Aging, 15(4), 51-68.

Rose, A. (1965). Older people and their social worlds : the subculture of the aging: a framework for research in social gerontology. Philadelphia: F. A. Davis.

Sharon, S. R. (2000). Successful aging in women (Thèse de doctorat inédite). The Union Institute Graduate College Stan Searl, United States of America.

Shin, K. R., Kim, M. Y., \& Kim, Y. H. (2003). Study on the lived experience of aging. Nursing and Health Sciences, 5, 245-252.

Signaté, A. (2006). Étude socioanthropologique du vieillissement en milieu rural à Rao département de Saint-Louis (Thèse de doctorat inédite). Université Cheikh Anta Diop de Dakar, Sénégal.

Tall, A. (2002). Les personnes âgées au Sénégal. Communication présentée à la deuxième Assemblée mondiale sur le vieillissement, Madrid, Espagne.

Van-Manen, M. (1990). Researching lived experience : human science for an action sensitive pedagogy. Ontario : The Althouse Press.

Awa Seck est professeure à l'École nationale de développement sanitaire et social (ENDSS) à Dakar au Sénégal. Ses champs d'intérêt couvrent la santé mondiale, la promotion de la santé, la recherche qualitative, le vieillissement des femmes, l'élaboration, la gestion et l'évaluation des programmes d'enseignement des soins infirmiers et des programmes de santé communautaire.

Ginette Lazure est professeure à la faculté des sciences infirmières de l'Université Laval à Québec et responsable du volet international. Ses champs d'intérêt portent sur la santé des femmes en contexte de pays en émergence, le développement de la compétence culturelle des professionnelles de la santé et la discipline infirmière. 
Diane Morin est professeure ordinaire à la Faculté de biologie et de médecine de l'Université de Lausanne en Suisse et y est directrice de l'Institut universitaire de formation et de recherche en soins (IUFRS). Elle est également professeure titulaire à la Faculté des sciences infirmières de l'Université Laval. Ses domaines de recherche comprennent l'évaluation des pratiques, la qualité des soins, la pratique infirmière avancée et les méthodes de recherche qualitatives et quantitatives. 